\title{
A score to settle-measuring Crohn's disease activity
}

Over the years, the Crohn's disease activity index (CDAI) has emerged as the gold standard for assessing disease activity in patients with Crohn's disease. However, along with other clinical indices and scores, the CDAI has some important limitations, notably that $\sim 40 \%$ of the index is derived from three subjective criteria (diarrhoea, abdominal pain and sense of well-being) whilst objective measures of inflammation (such as mucosal healing and C-reactive protein [CRP] levels) are not included. Thus, some clarity is needed on the best way to measure Crohn's disease activity.

Laurent Peyrin-Biroulet and colleagues have now conducted a retrospective analysis to investigate the relationships between clinical disease activity, normalization of CRP levels and mucosal healing in Crohn's disease. 188 patients from the SONIC (Study of Biologic and Immunomodulator Naive Patients in CD) trial were included in the study. These patients had evidence of mucosal ulcerations at baseline and had evaluable ileocolonoscopy and available CDAI and CRP values at week 26 of the study.

$53 \%$ of patients who had a CDAI $<150$ at week 26 achieved mucosal healing. The positive predictive value and negative predictive value of CDAI (using 150 as the cut-off value) to detect mucosal healing and normalization of CRP level were $79 \%$ and $42 \%$, respectively. "The accuracy of CDAI to predict the disappearance of objective signs of inflammation is low," concludes Peyrin-Biroulet. "This disconnect was poorly demonstrated in the IBD literature, even though all clinicians know that it exists."

The work has some important implications both for clinical trials and clinical practice. "We need a composite end point for clinical trials known as 'deep remission' if we want to assess drug efficacy using an objective evaluation," explains Peyrin-Biroulet. "Furthermore, when we see a patient during a clinical visit, evaluating clinical remission alone is not satisfactory; we should evaluate disease activity at the intestinal level by performing imaging studies, endoscopy and/or measuring calprotectin levels."

Isobel Leake

Original article Peyrin-Biroulet, L. et al. Clinical disease activity, C-reactive protein normalisation and mucosal healing in Crohn's disease in the SONIC trial. Gut doi:10.1136/ gutjnl-2013-304984 